\section{Design of paradise}

\author{
A Magic Web \\ by Egbert Giles Leigh Jr \& \\ Christian Ziegler \\ Oxford University Press: 2003. 292 pp. $\$ 40$
}

\section{Lawren Sack and Dina Dechmann}

Tropical rainforests are the settings for innumerable interactions between millions of organisms, a centrepiece of evolution. A Magic Web showcases the complexity of these interactions, using the rainforest that has been studied more than any other in the world, that of Barro Colorado Island (BCI), a $15-\mathrm{km}^{2}$ island in the Panama Canal.

Its author, Egbert Leigh, has worked on BCI since the 1960 s, and is especially concerned about what creates and supports tropical diversity. Here he summarizes data collected by hundreds of investigators, and argues that the finely tuned web of interactions between organisms is an important basis for the species diversity and complexity of tropical rainforest. Leigh points to many differences among species that are driven by direct competition. He argues convincingly for an equal importance of interactions between animals and plants. Plants diversify in their ways of escaping herbivores and enticing animals to pollinate their flowers and disperse their seeds. Animals diversify in the way they subsist on plants and avoid being eaten by other animals. It is a dynamic web; changes in climate can alter plant processes, with knock-on effects on the food available to animals. Loss of species, often induced by humans, unravels the web.

Leigh's wide-ranging vision truly comes alive when it meshes with Christian Ziegler's striking images. Ziegler has a degree in tropical biology and spent 15 months on the island photographing for this book. His collection of the typical as well as exceptional sights of BCI reveals a great intimacy with the forest. Ziegler does not simply illustrate the science, but rather illuminates each topic discussed, leading to a strong sense of the interactions everywhere in the jungle. Highlights include the sections on forest light gaps and plant diversity, on ant colonies, and on animal mimicry and camouflage.

The book will appeal to a broad audience. As a coffee-table book, it is enjoyable for its photographs and captions alone. The main text provides a useful introduction to tropical rainforest, surveying a wide range of ecological concepts - although, of necessity, briefly and in simple terms. These concepts are grounded with entertaining metaphors from human society. There are numerous comparisons with other ecosystems, and 'back of envelope' calculations. Ultimately, the book will enrich any scientist's view of biology. The tropical biologist will crave returning to the field.

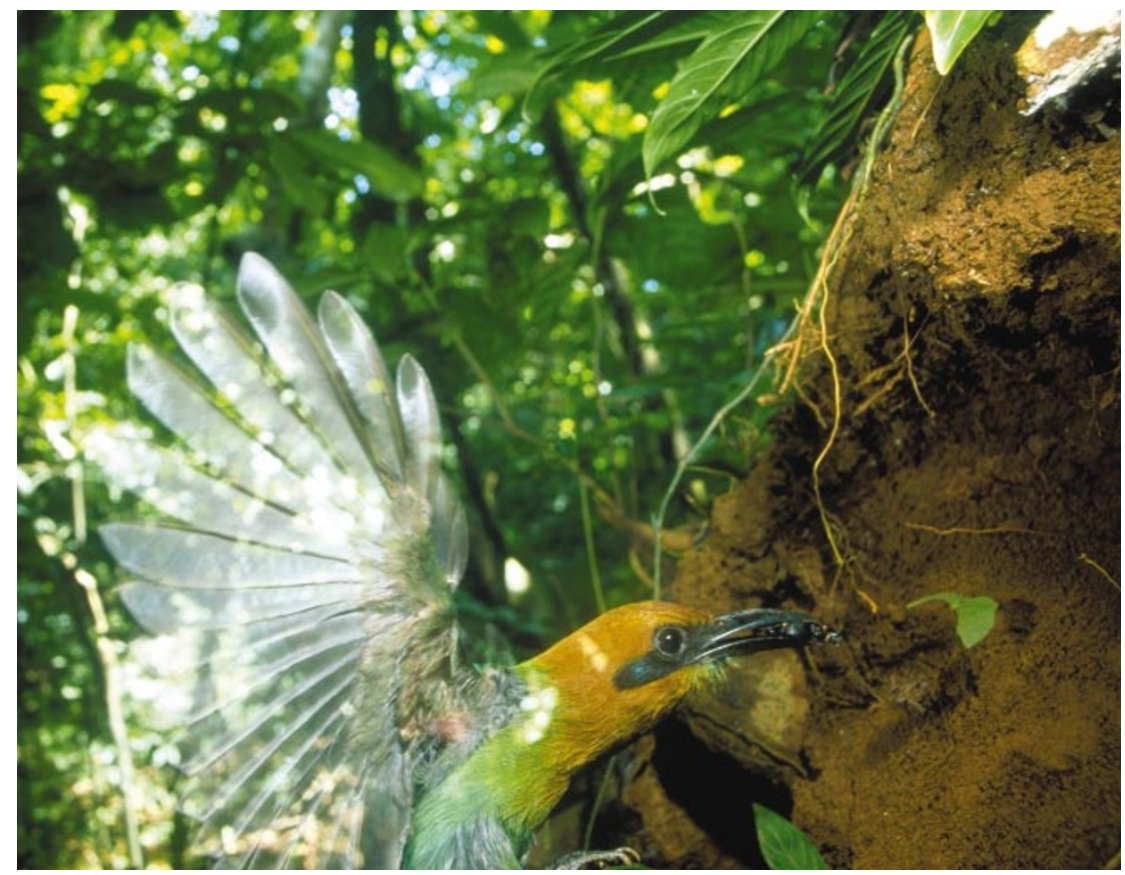

Forest food: a broad-billed motmot arrives home bearing an unfortunate beetle for its chicks' dinner.

Of course, even such a detailed treatment can be only a rough sketch of the intricate network of tropical-rainforest life. Leigh jokes that humans are not distinguished for their omniscience, and Ziegler reminds us how elusive are the visions of nature that he depicts. To fathom the web in all its detail, we will need to step up collaborations between scientists with diverse expertise and approaches. BCI, with its terrific facilities, has helped to

\section{Sex on the brain}

Essential Difference: Men, Women and the Extreme Male Brain/ Essential Difference: The Truth About the Male and Female Brain by Simon Baron-Cohen

Allen Lane: 2003. 256 pp. £16.99/

Perseus: 2003. 256 pp. $\$ 26$

\section{Joyce F. Benenson}

How male and female brains adapted differently to the environment in which humans evolved is an intriguing question. The traditional response from evolutionary scientists is that females' greater childcare responsibilities and males' greater involvement in intra-group and inter-group competition for resources and mates produced adaptations specific to these domains.

Baron-Cohen boldly suggests broader adaptations. Forming the foundation of his theory are his rich descriptions of Asperger's syndrome, a condition that affects more males than females by a ratio of up to $10: 1$. Individuals with Asperger's syndrome, build many bridges. Leigh and Ziegler's colourful synthesis should inspire many more to the task.

Lawren Sack is in the Department of Organismic and Evolutionary Biology, Harvard University, 16 Divinity Avenue, Cambridge, Massachusetts 02138, USA.

Dina Dechmann is in the Department of Animal Behaviour, University of Zurich,

Winterthurerstrasse 190, 8057 Zurich, Switzerland.

which is a mild form of autism, experience difficulties forming social relationships and communicating with others, and exhibit rigid and repetitive behaviours. Baron-Cohen interprets the symptoms of Asperger's syndrome as indicating an overabundance of systemizing and a lack of empathizing skills. On the basis of his clinical work with individuals with this condition, as well as his empirical research with unaffected children and adults, he infers that sex differences between typical males and females go beyond differential investment in children versus mating or hunting or warfare. Instead, he proposes that the male brain is programmed to systemize and the female brain to empathize, and that Asperger's syndrome represents the extreme male brain.

The book is written for a general audience, and it is easy and enjoyable to read. Case studies are intertwined with more systematic analyses. However, it is not the place to look for a comprehensive overview of empirical research in the field of either sex differences in personality or Asperger's syndrome. Because of the novelty of his ideas, BaronCohen defines his constructs on the basis of 
scales he has created and generously included in their complete forms in the appendices. Consequently, each reader can determine his or her own systemizing quotient, empathy quotient and autism quotient, as well as estimate those for one's family members, friends and less-liked associates.

The idea that males are more interested in systemizing than females merits serious consideration. Is there a common underpinning to such diverse pursuits as generating taxonomies of plants and animals, throwing balls or darts, skiing, figuring sports averages, creating maps and charts, designing buildings, repairing automobiles and houses, generating art, concocting culinary dishes, playing instruments, and studying mathematics, physics and engineering? Could it be that males engage in these behaviours more than females because of some heightened attraction to hypothesis testing and classificatory schemes? The question delves into the very nature of human intelligence itself. Because males typically ventured farther from their homes than females did in the environment in which humans evolved, those males who were able to systemize with greater precision might have gained an evolutionary advantage. Females who were more engaged than males in interaction with immediate family members in familiar environments might not have benefited so strongly from this type of skill. It is unquestionably a novel and fascinating idea that seems likely to generate a rich empirical body of literature as its properties are tested.

The second part of the theory - that females are more empathic than males — is more problematic. Baron-Cohen believes that females are better than males at caring about and communicating with others that is, at reading others' emotional cues and responding appropriately. To support this part of his theory, Baron-Cohen cites studies showing that, beginning in childhood, compared with males, females make more eye contact, converse more politely, are less overtly competitive, engage in less physical aggression, and as adults, commit fewer homicides and care more for children and other helpless individuals.

Other measures, however, show that males are highly socially skilled. Developmental research shows that, beginning in middle childhood, males are more likely than females to cooperate in same-sex groups and to include newcomers. Furthermore, despite the lower verbal intimacy between males than among females, males' friendships endure for longer. Anthropological studies show that as adults, males are more likely than females to forge strong political, military, financial, educational and religious alliances. These relationships require both a high level of investment in others as well as excellent communication skills, including the ability to decipher subtle

\section{Optical alchemy}

\section{Bridget Riley's constructions create ripples in the flat world of geometry.}

\section{Colin Martin}

For 40 years, Bridget Riley, pioneer of 'op art' (optical art) has used simple geometric shapes to make complex, sometimes visually unsettling paintings. As viewers' eyes move across a typical canvas its lines, spots, circles and squares - they perceive movement. Adjacent areas advance and recede.

Rather than using optical theory, Riley creates these visual effects intuitively. She refines her preparatory drawings until she achieves her desired result, which is to evoke emotion through formal abstraction. When satisfied, she entrusts the painting of finished canvases to studio assistants.

Her account of altering a single variable, the width of a square - in Movement in Squares (1961, right) - is reminiscent of experimental science. "I took a simple element [and] decided to change one of its characteristics, leaving the others intact. The result is a dramatic increase in tension. As the square comes under extreme pressure, a tremendous visual vibration is set up. In this critical area the contrast between blacks and whites is intensified and dazzling."

Movement between repose to disturbance is a constant feature in Riley's early, black-and-white work. Subsequently, she introduced colours using straight and then curved lines - producing the chromatic fields that result from interactions between colours. Throughout her career, Riley has returned to her earlier themes, as composers do in

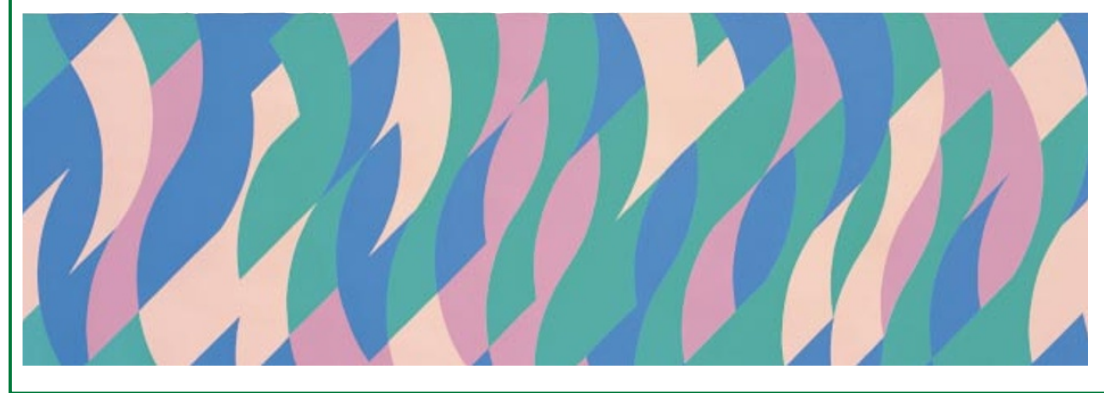

emotional signals and to respond appropriately. The fact that males are less verbally polite, discuss fewer personally intimate topics, and engage in more overt competition and aggression to achieve personal goals does not preclude them from forming strong bonds. On the contrary, males respect and desire to associate with other males who are powerful. My own research indicates that females and males excel at different types of social skill.

The link between Asperger's syndrome and the male brain, then, may work well for systemizing skills. However, the lack of interest in social relationships demonstrated by these individuals is inconsistent with the writing music. The beautiful rhythm that unfolds across 2003 canvas Evoë 3 (bottom), in which she sets curved shapes in pink and magenta against a blue and green ground, recalls the lyrical movement of Matisse's dancers.

Colin Martin is at 32 Woodstock Road, London W4 1UF, UK.

A retrospective exhibition of Bridget Riley's work can be seen at Tate Britain until 28 September 2003.

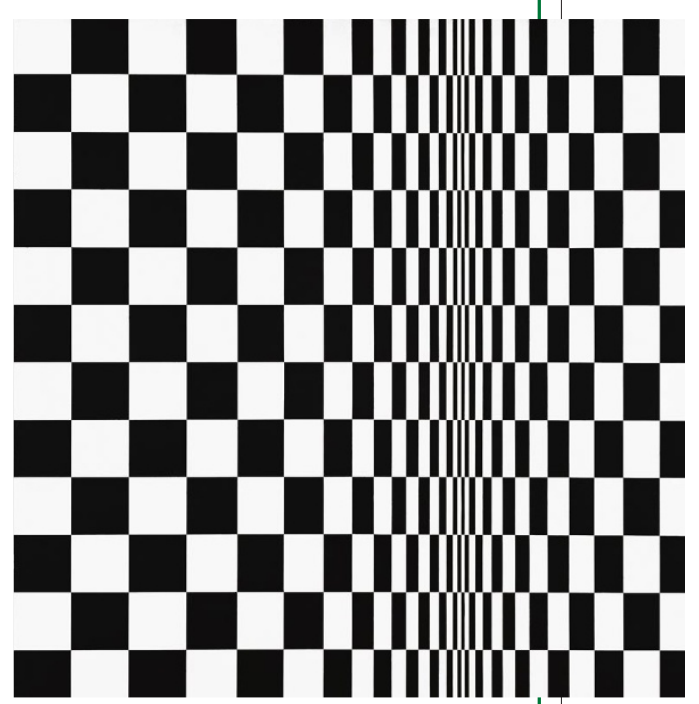

high level of social skills displayed by many males. Perhaps Baron-Cohen will find that individuals with Asperger's syndrome engage in the types of social skill displayed more by males than by females. If not, individuals with Asperger's syndrome might represent only one form of the extreme male brain. Another form would include males who score highly on systemizing skills and who are also highly sociable. Regardless of the ultimate answer, this book inspires the reader to reconsider traditional assumptions about the skills of each sex.

Joyce F. Benenson is in the Department of

Psychology, University of Plymouth, Drake Circus, Plymouth, Devon PL4 8AA, UK. 